\section{Significance of patient positioning guidelines in endoscopic procedures}

Patients are placed on an
operating table for surgery
or, in the event of endloscopic
treatment, on a trolley. Surgery
may need patient placement
that is not at all comfortable,
posing challenges for the
patient. There are well-
established rules for patient
positioning during surgical
operations, but tho such criteria
are available for endoscopic
treatments. A study conducted
by Dr Vera Meeusen and her
team from Princess Alexandra
Hospital, University of
Queensland, demonstrates it is
possible to apply most of the
patient-positioning guidelines
for surgical procedures to
gastrointestinal endoscopic
procedures in endoscopy units.

urgery may need patient comfortable, posing challenges for the patient. Patients may experien muscles lying on som ovistretched against something sharp. They can also experience overstretching or compressing nerves and squeezing skin tissue, which can result in temporary or permanent injuries/damage. Patient positioning (PP) involves placing the patient on the operating table (or trolley) in such a way that the doctor can complete the procedure while the patient remains comfortable. There are well-established rules for PP during surgical operations, and nurses and surgeons are well-versed in how to follow them

Unfortunately, endoscopic treatments do not have these criteria. Endoscopy body are looked at using niside the called an endoscope. Gastrointestinal endoscopists and endoscopy nurses do not receive training in patient positioning safety. DrVera Meeusen and her team from Princess Alexandra Hospital, University of Queensland, have tested whether the existing guidelines of patient positioning can be applied to the endoscopic procedures. Correct posture seeks to ensure safety and efficiency subsequent wellbeing such as limiting the risk of nerve and tissue damage. THE NEED FOR PATIENT POSTONING IN ENDOSCOPIC PROCEDURES
Traditionally, gastroenterologists in sedation or without any patient sedation. The majority of the caseload consists of diagnostic gastrointestinal minutes on operatic sthat last 30-60 why in contrast to surgical procedures performed in the operating room, patient placement has received little attention in endoscopy. Advanced endoscopic interventions (eg, peror endoscopic myotomy and endoscopic sleeve gastroplasty) have become established in expert centres over the last few decades, with procedure times exceeding 60 minutes and in some cases lasting several hours, and frequently requiring general anaesthesia. Peroral endoscopic myotomy (POEM) is a minimally invasive endoscopic procedure intended for long-term achalasia (benign motility disorder). Endoscopic sleeve gastroplasty is a loss procedure.

Obese patients are more likely to have medical conditions such as narrowing of the arteries), arthritis, sleep apnea syndrome (a potentially serious sleep disorder in which breathing repeatedly stops and starts), alveolar hypoventilation (a rare condition in which a person does not take enoug breaths per minute), urinary stress incontinence (unintentional loss of urine), and gastroesophageal reflux containing contents in your stomach persistently leak back up into your oesophagus, the tube from your throet endoscopic operations under procedura recovery from symptoms of oesophaga disease (a condition in which acid-
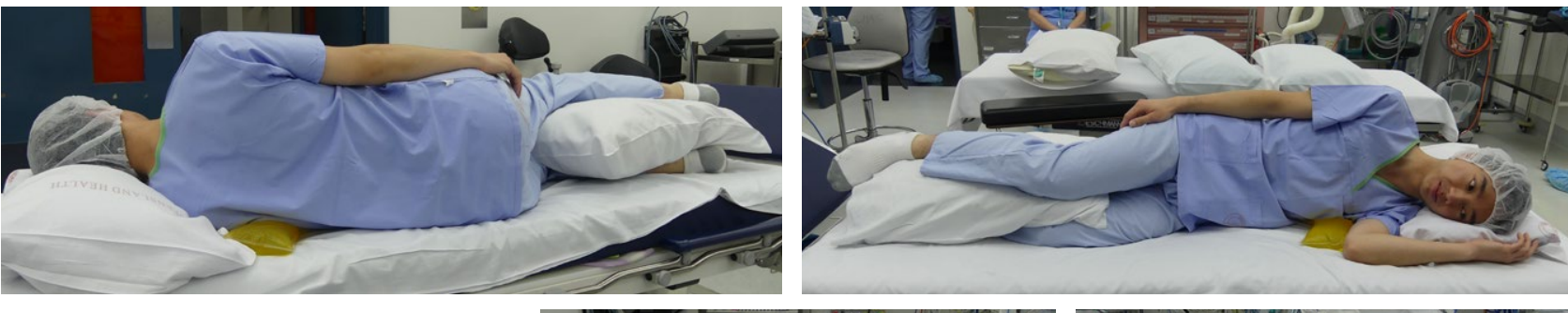

Patient positioning in the lateral position can be
challenging due to a lack of competency and suitable positioning devices.

increase the risk of patient injuries, educe tissue perfusion (blood flow), and equipment is required for these patients (eg, sideboards attached to the operating table, extra-wide and extralong safety belts, a ramping posture, r capable of weight support).

ENDOSCOPY UNITS ARE FACING NEW CHALLENGES

The proper posture of surgical patients in an operating theatre has garnered a lot of attention for a long time. Surgical practice guidelines are widely available, and anaesthesia and perioperative nursing professionals receive instruction in patient posture as part of their education. The goal of the recommendations is to avoid exposing body surfaces to too high and prolonged pressure, which is the underlying concept that leads to pressure injuries. This can be performed by using pressure-relieving beds or cushions, as well as proper patient positioning. Evidence reveals prone positions can be in lateral and due to a lack of competency amo nurses and porters, as well as a lack of suitable positioning devices. Despite the widespread availability of patient positioning guidelines for surgical procedures, neither the America Society of Gastroenterology Nurses and Associates, the Gastroenterological Nurses College of Australia, nor the European Society of Gastroenterology Nurses and Associates mentions pati positioning during gastrointestin endoscopic procedures in their standards, guidelines, or publications.

During endoscopic operations, the supine, left lateral, and prone positions are currently the prefered methods of

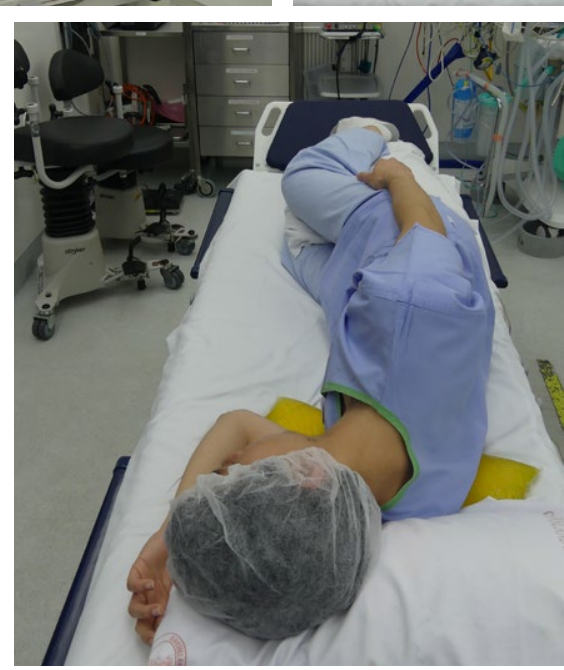

is used in the majority of surgical procedures. A range of surgical procedures, including thoracic, hip, and lateral position. Several forms of surgery,

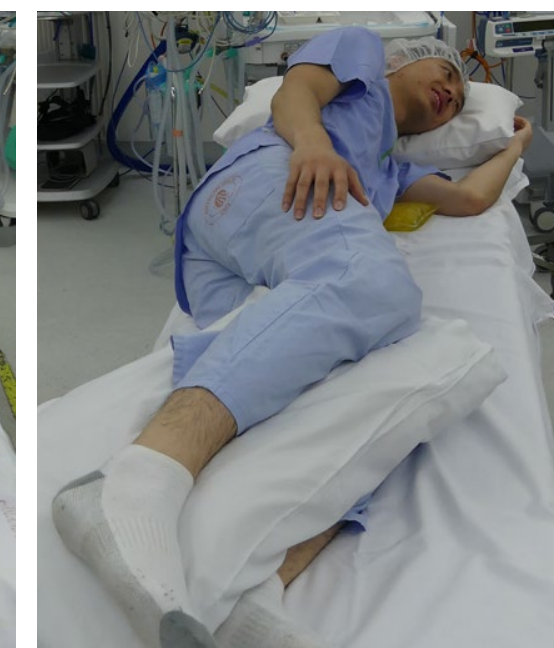
including brain and spinal surgery, are

llowing the endoscopist to face the patient directly. Endoscopy units are acing new challenges as the number of complex and lengthy advanced procedures performed on patients with

Endoscopy units are facing new challenges as the number of complex and lengthy advanced procedures performed on patients with comorbidities and obesity rises.

performed in the prone position

However, depending on the pressure used, these positions cance measures injury, skin damage, or compression of tissue or vascular systems, resulting in localised ischemia - a condition where parts of the body aren't getting enoug blood (and thus oxygen). The increased occurrence of these injuries in patients with obesity and related comorbidities can increase the risk of these injuries even more. The prone position during endoscopic treatments has several advantages for the proceduralist and anaesthetic team, including reduced risk of aspiration, easier access to the positioning practice recommendations re therefore all the more important. AND COMFORT LEVEL The goal of DrVera Meeusen and her team's study was to see if patient placement standards for surgical operations in operating rooms might restop procedures without compromising safe prostigated (BMI $18.5 \mathrm{~kg} / \mathrm{m}^{2}$ ) tho mass index with a high BMI (BMI >35 kg/m²). Three volunteers were found among hospital BMI (BMI 18.5-24.9k k/2), and tho 


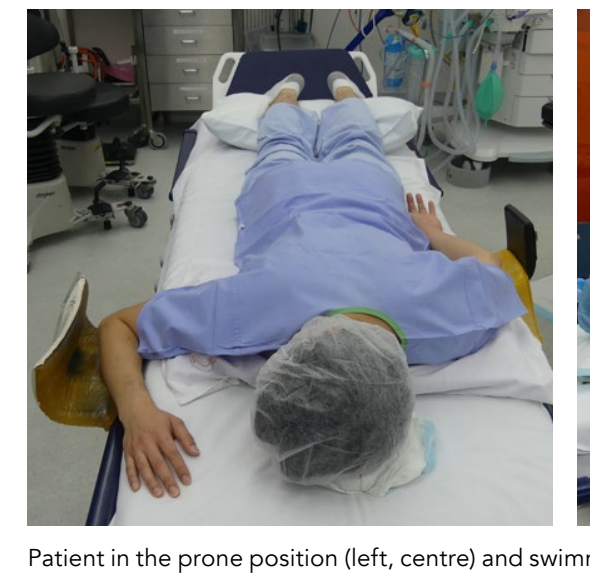

workers, one for each BMI group, with BMls of $18 \mathrm{~kg} / \mathrm{m}^{2}(\mathrm{male}), 23.5 \mathrm{~kg} / \mathrm{m}^{2}$
(female), and $43 \mathrm{~kg} / \mathrm{m}^{2}$ (female).

The research project was split into two parts. First, the volunteers were placed on the operation table in the recommended by best-protice petientplacement recommendations. Gel mats, gel rolls, cushions, and arm boards were used for safe positioning. Th participants were asked to rate their leve of comfort after they were positioned,

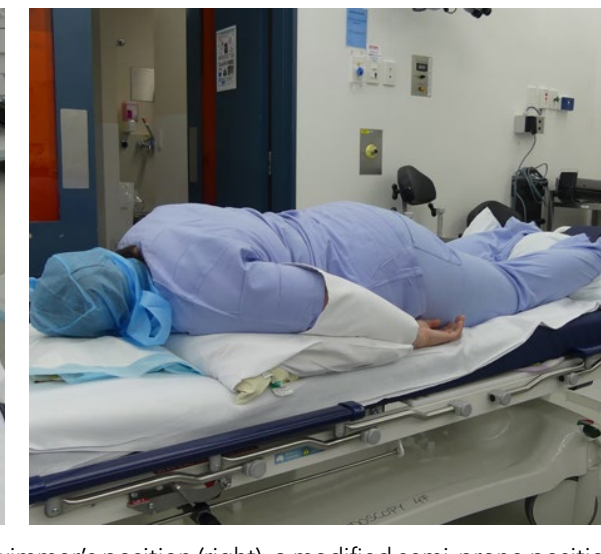

surgical operations in operating rooms. The level of comfort was measured on more, and photographs were taken the operating table and the endoscopic the operating table and the endoscopic strecher, however, he level of comtort BMl groups.

This study demonstrates that it is possible to replicate patient positioning guidelines for surgical procedures in in endoscopy units, except foroed

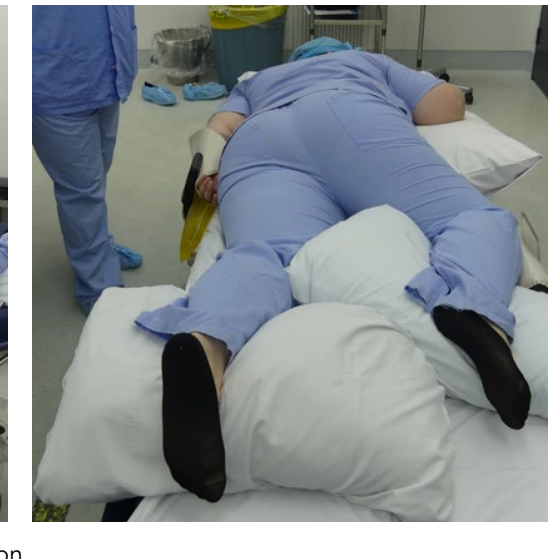

was the most difficult for endoscopists to work with. It was impossible to put the head in a neutral forward posture because it would restrict the endoscopist's access to the mouth. The head was put on the edge of the pillow access, while the height of the head was kept in line with the cenical spine. Volunteers felt most ease in the prone position with their head aligned with the cervical spine and slightly turned to the right, left arm next to the body, and the elbow of the right arm flexed with the and photographs of the actual positioning
were taken. Second, the volunteers were placed on the endoscopic patien stretcher in the
supine, lateral, and prone positions using positioning in lateral and prone Establishing positioning standards for of the shoulder endoscopic procedures in the supine, called swimmer's prone, and lateral positions is critical. position, a modified prone (semi-prone) the same recommendations. positions, and head positioning in the During the second phase, an prone position. Both supine and lateral posture were found to be equally comforable by all participants. When the endoscopic stretcher, the obe volunteer felt much less comfortable in altered according to the endoscopist's instructions, while still attempting to follow the patient positioning criteria for the prone position. The prone position

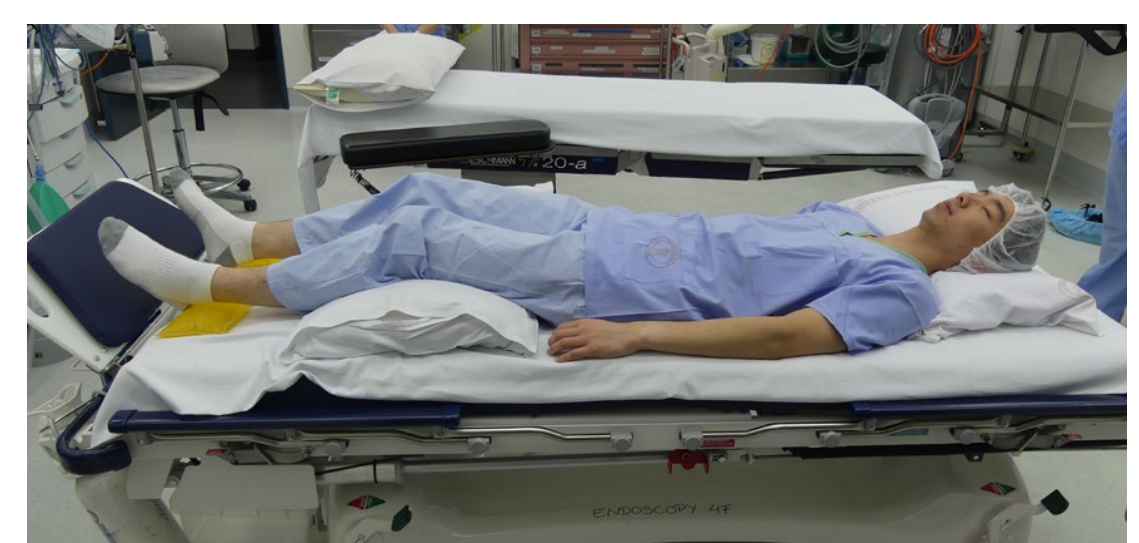

position, was also investigated. Placing cushions under the right side of the volunteer' thorax and abdomen, was the most pleasant choice.

\section{OOOKING AHEAD}

Establishing positioning standards the critical. The new guidelines for patient
chention placement during gastrointestinal endoscopic procedures should be included in national endoscopy practice standards and endoscopy nurse education. The present rules for patient positioning in operating rooms dunng surgical operations could be used as a guide. Real-world research with real-world patients during real-world endoscopic operations is required. More study is needed to establish a method to estimate the best size of gel roll for each
patient's rib cage in the lateral position.

\section{Behind the Research}

\section{DrVera Meeusen}

E: vera.meeusen@health.gld.gov.au T: +61731769170

\section{Research Objectives}

Dr Meeusen tested whether established guidelines Dr endoscopic procedures.

\section{Detail}

\section{Address}

Endoscopy Unit, Department of Gastroenterology PQ Woolloongabba

4102 Queensland, Australia

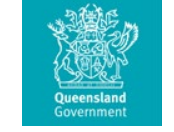

Vera Meeusen is a Clinical Nurse Consultant of the Endoscopy Unit at Princess Alexandra Hospital University of Queensland. She started as Nurse Anaesthetist and performed several clinical research projects. She obtained her PhD in Medicine in 2010. She continued working in the perianaesthesia and gastroenterology environment with an emphasis on

\section{Funding}

The Australian College of PeriAnaesthesia Nurses (ACPAN) provided a grant to support this study.

\section{Collaborator}

The study was performed with help of the anaesthesia

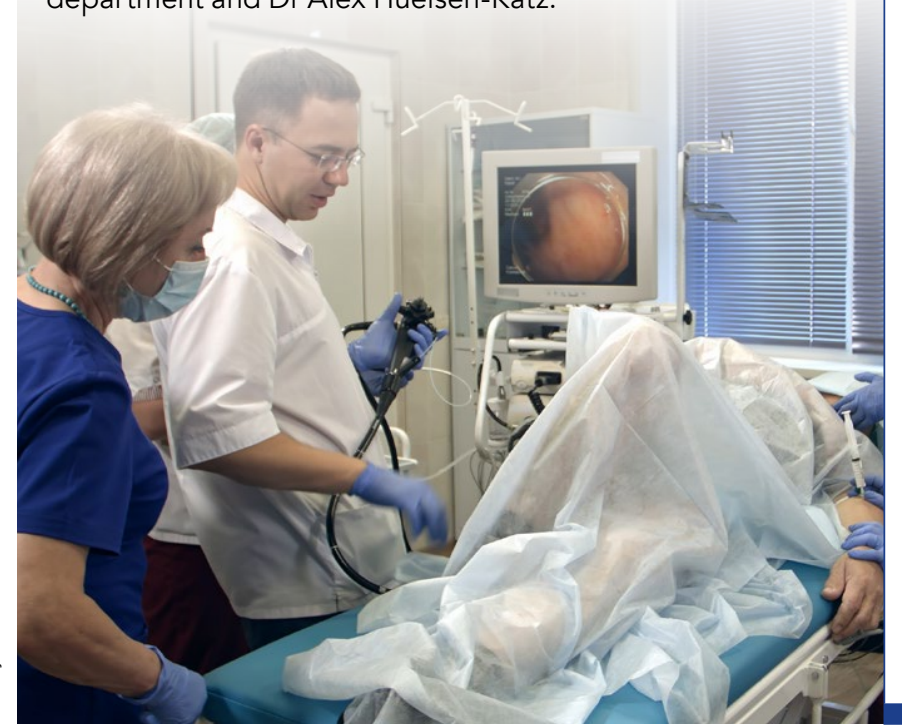

\section{References}

Meeusen, V, Kim, N, Elson, J, Ma, R, Newman, F, Bendeich, J, Holtmann, G, (2021) Patient Positioning Gastroenterology Nursing, 44(3), 185-191. doi.org/10.1097/ sga.00000000000000534

Meeusen, V, Rogue, M, Elson, J, (2019) Case report: Pressure injury after prolonged endoscopic intervention. GENCA Journal, 29(2), 12-14.

Sørensen, EE, Kusk, KH, Grønkjaer, M, (2016) Operating room nurses positioning of anesthetized surgical patients. Journal of Clinical Nursing, 25(5-6), 690-698.

Hartley, J, Baitch, L, (2015) Patient positioning during anaesthesia. Anaesthesia Tutorial of the Week, 311 www.wfsahq. org

Spruce, L, Van Wicklin, SA, (2014) Back to basics: positioning the patient AORN Journal, 100(3), 298-305.

\section{Personal Response}

What suggestions do you have for researchers investigating and trying to identify the most suitable patient position for gastrointestina endoscopic procedure?

IU - Most urgently is the optimisation of the prone position. We have recently finalised a study regarding the prone and semi-prone positioning during ERCP procedures.

- More research is needed on whether there is a need for special gel mats/pillows to improve ora access and positioning of the arms.

Further research is also needed to establish safe work instructions in how to apply patient positioning without the requirement of physical heavy manual handling by healthcare practition Aids used in theatre to transfer patients from bed/trolley to operating table are not suitable or are lifted by nurses and porters (especitily obese patients). 